\title{
Revisiting 3D van Krevelen diagrams as a tool for the visualization of volatile profile of varietal olive oils from Alentejo region, Portugal
}

\author{
Nuno Martins ${ }^{\mathrm{a}, \mathrm{b}, 1}$, Nicasio T. Jiménez-Morillo ${ }^{\mathrm{b}, \mathrm{c}, 1}$, Flávia Freitas ${ }^{\mathrm{d}}$, Raquel Garcia ${ }^{\mathrm{b}}$, \\ Marco Gomes da Silva ${ }^{\mathrm{d}}$, Maria João Cabrita ${ }^{\mathrm{b}, \mathrm{e}, *}$ \\ ${ }^{a}$ Aix Marseille Univ, Univ Avignon, CNRS, IRD, IMBE, Marseille, France \\ ${ }^{\mathrm{b}}$ ICAAM - Instituto de Ciências Agrárias e Ambientais Mediterrânicas, Universidade de Évora, Núcleo da Mitra, Ap. 94, 7006-554, Évora, Portugal \\ ${ }^{\mathrm{c}}$ HERCULES, Universidade de Évora, Palácio do Vimioso, 7000-089, Évora, Portugal \\ ${ }^{\mathrm{d}}$ LAQV-REQUIMTE, Departamento de Química, Faculdade de Ciências e Tecnologia, Universidade Nova de Lisboa, 2829-516, Caparica, Portugal \\ ${ }^{\text {e }}$ Departamento de Fitotecnia, Escola de Ciências e Tecnologia, Universidade de Évora, Núcleo da Mitra, Ap. 94, 7006-554, Évora, Portugal
}

Keywords:

Volatile compounds

Monovarietal olive oils

HS-SPME-GC/MS

Chemometric analysis

3D van Krevelen diagram

\begin{abstract}
This paper aims to study the volatile composition of monovarietal extra-virgin olive oils from different varieties from Alentejo region (Portugal). Volatile profile of 82 olive oils was performed by head-space solid phase microextraction hyphenated with gas chromatography/mass spectrometry (HS-SPME-GC/MS). A total of 107 volatile compounds, belonging to several class of compounds, like aldehydes, hydrocarbons, alcohols, terpenoids, ketones, sulphurous compounds, acids and esters were identified, assigned to a specific biogenic origin and total abundances were plotted in modified 3D van Krevelen diagrams. The 3D plots showed that there are compositional differences among olive oil varieties. Discriminant analysis confirmed that the pattern of volatile compounds includes enough information to group sample variety amongst discrete monovarietal olive oil. Combining statistical analysis (t-Student) and graphical-tools (van Krevelen diagram) seems to be a powerful tool to determine what molecular families were characteristic of each olive oil variety, contributing to the valorization of monovarietal Portuguese olive oil.
\end{abstract}

\section{Introduction}

Olive oil is the most consumed vegetable oil in the Mediterranean basin due to the health benefits, nutritional properties and peculiar organoleptic characteristics, which are closely related to their highly complex chemical composition. Recently, it has been ascribed potential therapeutic effects to some of those compounds [1]. Thus, olive oil is nowadays considered as a functional food, arousing a huge interest for its consumption. Olive oil is mainly composed of triacylglycerols, free fatty acids, mono and diacylglycerols, hydrocarbons, sterols, aliphatic alcohols, tocopherols and pigments, as well as various phenolic and volatile compounds [2,3]. Most of the volatile compounds are formed during the mechanical extraction process encompassed on olive oil production, through the action of enzymes that are released when the fruits are crushed, and continue to form during malaxation by means of enzymatic reactions, known as lipoxygenase pathway. It is well known that the endogenous plant enzymes, through the lipoxygenase pathway, are involved on the formation of volatiles related to positive aroma perceptions of olive oil. Whereas the occurrence of sensory defects is promoted by the microbial activity resulting from chemical oxidation and exogenous enzymes. Volatile compounds, mainly produced by the oxidation of fatty acids, contribute to the organoleptic characteristics of olive oil and are essential for their sensorial quality [4-6]. Several studies reported that the aroma of olive oil is attributed to aldehydes, esters, alcohols, ketones, hydrocarbons, furans and, eventually, other yet unidentified volatile compounds $[7,8]$. The major volatile compounds reported in extra-virgin olive oils (EVOO) are the $\mathrm{C}_{6}$ and the $\mathrm{C}_{5}$ volatile compounds. The pleasant notes in olive oils is due both to $\mathrm{C}_{6}$ aldehydes, alcohols, and their corresponding esters, and to minor amounts of $\mathrm{C}_{5}$ carbonyl compounds, alcohols, and pentene dimers [9].

The distinctive EVOO aroma consists of a complex mixture of volatile compounds, which is strongly variable in function on olive fruit composition, which depends on the cultivar, the ripening stage and the environmental growth conditions, such as biotic and abiotic stresses 
Table 1

Cultivar, region, geographic information and annual average precipitation and temperature of different EVOOs.

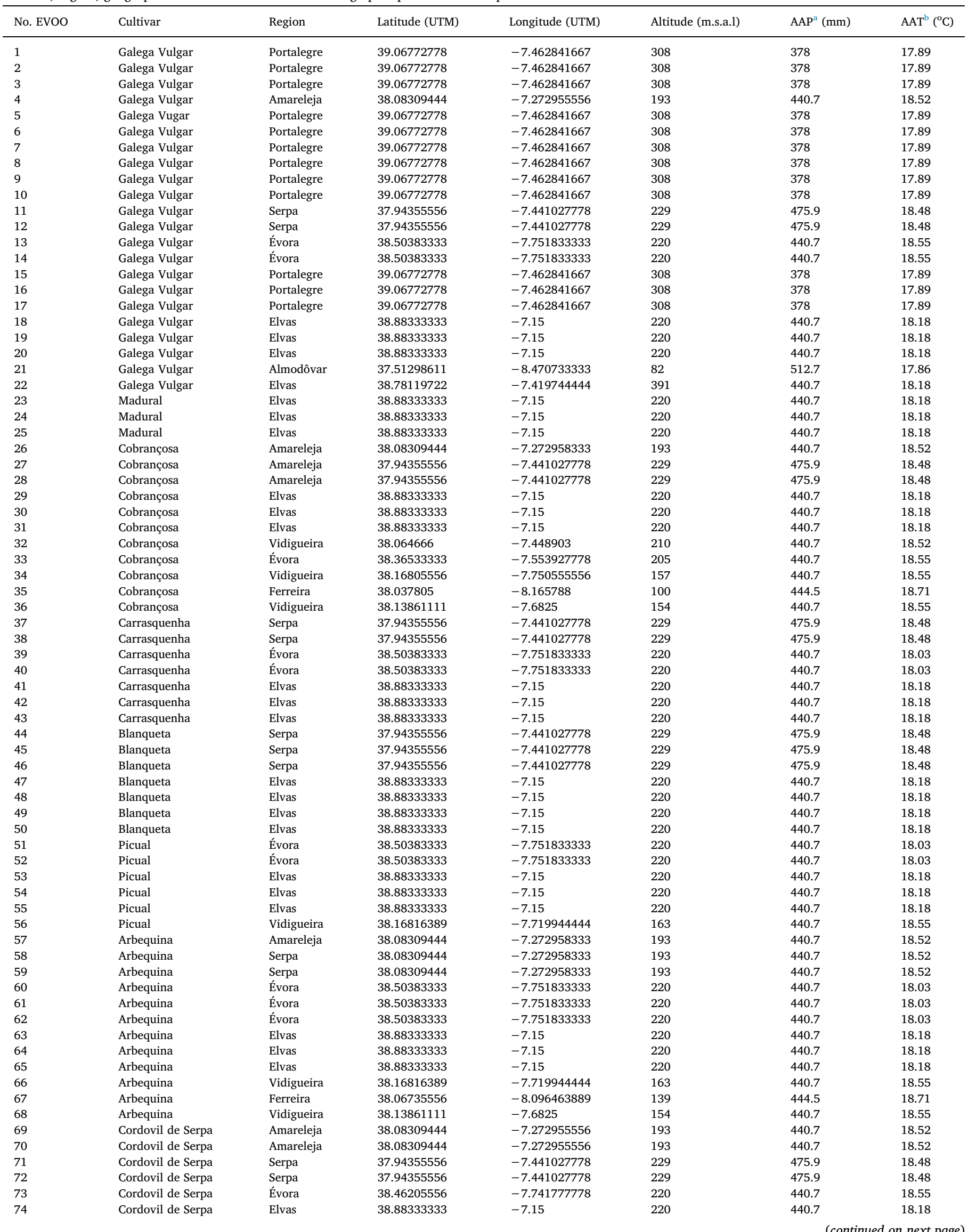


Table 1 (continued)

\begin{tabular}{|c|c|c|c|c|c|c|c|}
\hline No. EVOO & Cultivar & Region & Latitude (UTM) & Longitude (UTM) & Altitude (m.s.a.l) & $\mathrm{AAP}^{\mathrm{a}}(\mathrm{mm})$ & $\operatorname{AAT}^{\mathrm{b}}\left({ }^{\mathrm{O}} \mathrm{C}\right)$ \\
\hline 75 & Cordovil de Serpa & Elvas & 38.88333333 & -7.15 & 220 & 440.7 & 18.18 \\
\hline 76 & Cordovil de Serpa & Elvas & 38.88333333 & -7.15 & 220 & 440.7 & 18.18 \\
\hline 77 & Cordovil de Serpa & Vidigueira & 38.064666 & -7.448903 & 210 & 440.7 & 18.52 \\
\hline 78 & Verdeal Alentejana & Serpa & 37.94355556 & -7.441027778 & 229 & 475.9 & 18.52 \\
\hline 79 & Verdeal Alentejana & Serpa & 37.94355556 & -7.441027778 & 229 & 475.9 & 18.52 \\
\hline 80 & Verdeal Alentejana & Elvas & 38.88333333 & -7.15 & 220 & 440.7 & 18.18 \\
\hline 81 & Verdeal Alentejana & Elvas & 38.88333333 & -7.15 & 220 & 440.7 & 18.18 \\
\hline 82 & Verdeal Alentejana & Elvas & 38.88333333 & -7.15 & 220 & 440.7 & 18.18 \\
\hline
\end{tabular}

a Annual average precipitation.

b Annual average temperature.

[10]. The technological features of the milling process and olive oil storage conditions also have a strong impact in the volatile composition $[11,12]$. In the last years, autochthonous monovarietal olive oils were the focus of interest of producers. Thus, the development of analytical tools that enable to distinguish them, highlighting the peculiarities of each variety is highly warranted allowing to valorize EVOO. Indeed, consumers are also more and more oriented towards purchasing food products of a certified genuineness and geographical origin, and for olive oil matrices this topic is also emerging in the last years. Thus, the characterization of the chemical composition of monovarietal EVOO from a specific production area is crucial. There are some scientific works that try to characterize some cultivars $[5,13,14]$, but in fact, for the Portuguese varieties there is still a lack of information about their volatile composition. In order to contribute to overcome this gap, in this work is attempted the characterization of the volatile composition of monovarietal EVOO produced with different varieties of olives from the Alentejo region.

To achieve this main goal, a sample preparation methodology is required in order to pre-concentrate/isolate the target analytes. This is imperative in high complex food samples like olive oil. Solid phase microextraction (SPME) has proven to be a powerful technique providing many advantages over conventional sample preparation techniques. It is simple to use, fast and inexpensive, requires less sample preparation time and handling, does not require solvent extraction, and allows characterization of head-space (HS) in contact with the sample, resulting in cleaner chromatograms [15]. In particular, HS-SPME technique has proved to be extremely efficient as sample preparation methodology in the volatile characterization of different matrices with high complexity, such as olive oil [7], enabling an effective isolation/ pre-concentration of the aroma compounds, that will be further analyzed using GC/MS technique.

However, data obtained by chromatographic techniques such as GC/MS can be complex and their interpretation very demanding. Therefore, the use of multivariate statistical analysis is required to achieve a correct interpretation of chemical data, as well as the chemical profile of different varieties of olive oils [16]. Several studies have shown that volatile compounds can be employed as fingerprint to assess traceability and authentication of olive oil samples in tandem with several chemometric tools like analysis of variance (ANOVA) and correlation analysis [5], principal component analysis (PCA) [17] and linear discriminant analysis (LDA) [18]. The chromatographic data (relative abundance of compound-specific) can also be analyzed by graphic tools, such as the classic van Krevelen diagram [19]. This graphical tool has the advantage of showing the density (chromatographic area) of different regions of the $\mathrm{H} / \mathrm{C}$ vs. O/C surface, facilitating the comparison among samples. Since this representation appears well suited to amplifying and exposing compositional differences within and between complex samples, this work aims also to explore the usefulness of this tool to discriminate olive oil varieties from Alentejo region based on their volatile profile. Traditionally, this diagram was intended to assess the coal maturity degree by the comparison between $\mathrm{H} / \mathrm{C}$ and $\mathrm{O} /$ $\mathrm{C}$ ratios of samples. Nowadays, an updated surface density van Krevelen graphical statistic method, is used to handle a large chemical data matrices, such as analytical pyrolysis or ultra-high resolution mass spectrometry [20-23]. This method may also represent statistical indices calculated by uni- or multivariate analysis [23], as well as the subtraction values among compounds generated by different chemical families. Within the present work, it was carried out a molecular and chemometric analysis of the chromatographic data obtained by HSSPME-GC/MS, from volatile compounds of different EVOOs produced from different varieties of Portuguese olives, in order to identify the molecular markers of each olive oil variety. Thus, this paper could be considered as a fundamental contribution to the characterization of the different monovarietal EVOOs from the Alentejo region of Portugal.

\section{Materials and methods}

\subsection{Samples and experimental design}

Olive fruits of nine varieties of Olea europaea L., (Blanqueta, Verdeal Alentejana, Madural, Picual, Arbequina, Cordovil de Serpa, Cobrançosa, Carrasquenha and Galega Vulgar) were sampled from different sub-regions of the Alentejo region of Portugal. The olives were harvested in November and December 2017 at the commercially ripe stage. For most of the samples, approximately $5 \mathrm{~kg}$ of each olive varieties was processed separately in an Abencor ${ }^{\circledR}$ system immediately after harvesting. Fruits were crushed with a hammer mill and the olive paste was malaxed at $25^{\circ} \mathrm{C}$, room temperature for $30 \mathrm{~min}$, in an olive paste mixer, finally the olive oil was separated by centrifugation. Additionally, some samples were processed by commercial olive oil mills. Samples were collected immediately after processing to avoid possible undeclared mixtures with oils from other cultivars and geographical origins before bottling. They were stored in dark-brown glass bottles at $20^{\circ} \mathrm{C}$ in the dark. Table 1 shows the detailed information about the different EVOOs studied in this work.

\subsection{Volatile extraction procedure}

A carboxen/divinylbenzene/polydimethylsiloxane fiber (CAR/DVB/ PDMS, $1 \mathrm{~cm}, 50 / 30 \mu \mathrm{m}$ film thickness (df)) supplied from Supelco, (Bellefonte, PA, USA) were used for HS-SPME extractions. Fiber blanks were run periodically to ensure the absence of contaminants and/or carryover. HS-SPME extraction was performed according to following procedure, based on Martins et al. [24] slightly modified: $4.0 \mathrm{~mL}$ of each olive oil sample and $0.6 \mathrm{~g}$ of sodium chloride were introduced in a $22.0 \mathrm{~mL}$ vial and sealed with a Teflon-lined rubber septum/magnetic screw cap, the vial was equilibrated for $10 \mathrm{~min}$ at $50{ }^{\circ} \mathrm{C}$ and then extracted for $50 \mathrm{~min}$ at the same temperature, and thermal desorption of the analytes was carried out by exposing the fiber in the GC injection port at $260^{\circ} \mathrm{C}$ for $3 \mathrm{~min}$ in splitless mode. 


\subsection{Chromatographic analyses}

The analyses were performed on a gas-chromatography/mass spectrometer (GC/MS) system consisting of a Bruker GC 456 with a Bruker mass selective detector Scion TQ. An automatic sampler injector was used: CTC Analysis autosampler CombiPAL. Data were acquired with MSWS 8.2 Bruker and analyzed with Bruker MS Data Review 8.0. Chromatographic separation was achieved on a ZB-WAX PLUS capillary column $(60 \mathrm{~m} \times 0.32 \mathrm{~mm}$ i.d., $1.0 \mu \mathrm{m} \mathrm{df})$ supplied by Phenomenex, Torrance, CA, USA. The oven temperature program began at $40^{\circ} \mathrm{C}$ hold for $5 \mathrm{~min}$, raised at $4{ }^{\circ} \mathrm{C} \mathrm{min}^{-1}$ up to $240{ }^{\circ} \mathrm{C}$ and hold for $5 \mathrm{~min}$. Helium was used as carrier gas constant pressure of 35.0 Psi at the Electronic flow control (EFC 21) and 23.0 Psi at the EFC 24. The MS transfer line and source temperatures were set at, respectively, $240{ }^{\circ} \mathrm{C}$ and $220^{\circ} \mathrm{C}$. Spectra were matched NIST MS Search Program Version $2.0 \mathrm{~g}$. To determine the retention times and characteristic mass fragments, electron ionization (EI) at $70 \mathrm{eV}$ mass spectra of the analytes were recorded at full scan, from 40 to $450 \mathrm{Da}$. The linear retention index values were calculated through analysis of the commercial hydrocarbon mixture $\left(\mathrm{C}_{8}-\mathrm{C}_{20}\right)$, using the same chromatographic conditions. The volatile compounds were identified by matching mass spectra with spectra of reference compounds in NIST mass spectral library, also taking into consideration structure and molecular weight, and additionally, by comparing retention times generated for each reference compound analyzed using a commercial hydrocarbon mixture $\left(\mathrm{C}_{8}-\mathrm{C}_{20}\right)$ for determination of the linear retention indices (LRI) in comparison with the LRIs described in the literature (Table S1 in supplementary material). The relative amount of each compound was calculated as the percent ratio of the respective peak area relative to the total peak area and expressed as percentage (\%).

\subsection{Graphical representation of chromatographic compounds}

Using the structural information provided by the GC/MS analysis it was possible to construct surface density van Krevelen plot. The compound-specific atomic ratios $(\mathrm{H} / \mathrm{C}$ and $\mathrm{O} / \mathrm{C})$ were calculated from the empirical molecular formulas inferred from the mass spectra. Only compounds identified with a peak area $>0.2 \%$ of the total chromatographic area were included in the analyses. An additional refinement for displaying mass spectrometry results is the building up 3D van Krevelen diagram, recently described in Almendros et al. [23]. In short, mass spectrometry data were represented by plotting chromatographic yields for individual compounds calculated as total abundances (z), as density surfaces in the $\mathrm{x}, \mathrm{y}$ plane defined by its atomic $\mathrm{H} / \mathrm{C}(\mathrm{y})$ and $\mathrm{O} / \mathrm{C}$ (x) ratios, as calculated from the empirical formulas. The surface density plots are made for average values of the chromatograms of different samples into the same olive variety (i.e. it is calculated the average value of abundance for each one of chemical compounds in each chromatogram). Furthermore, it is made a model sample with the average of all EVOO samples (mixing all olive oil varieties). Despite the limitations of surface plots for comparing multiple samples, the differences between pairs of samples may be also graphically displayed as subtraction surfaces with positive zone (blue) and negative zone (red), that illustrate the differences in proportion of a concrete family group of volatile compounds for each olive variety. By last, it was calculated for each chemical compound the t-Student value to know if the difference between subtracted chemical families has a signification higher than 95\% $(P<0.05)$. The chemical compounds significantly different $(P<0.05)$ are represented as a superposed contour van Krevelen diagram.

\subsection{Statistical analysis}

In order to analyse the molecular markers of volatile compounds of Portuguese EVOO samples from different olive varieties, chromatographic data were analyzed by linear discriminant analysis (LDA). This statistical technique is a multivariate classification tool, which rotates the original space, maximizing the separation between classes, while minimizing the distance between objects within the same classes. In this work, the classes correspond to the olive varieties. The abundances of the different compounds families of EVOO volatiles were used as independent variables. The LDA was processed using the "Statigraphics Centurion v. 15.0". In addition, Student's $t$ test of different family compounds was calculated using Microsoft Excel 2013.

\section{Results and discussion}

\subsection{Volatile profile of EVOOs of different cultivars}

This work reports the discrimination among different single-cultivar EVOO samples through HS-SPME-GC/MS analysis. Eighty two (82) monovarietal olive oils produced from 9 different varieties of olives (Blanqueta, Verdeal Alentejana, Madural, Picual, Arbequina, Cordovil de Serpa, Cobrançosa, Carrasquenha and Galega Vulgar) from the Alentejo region of Portugal were analyzed. In the volatile fraction of EVOOs analyzed, were identified and semi-quantified a total of 107 compounds belonging essentially to the chemical classes of the esters, alcohols, aldehydes, acids, ketones, linear and branched alkyl, sulphur

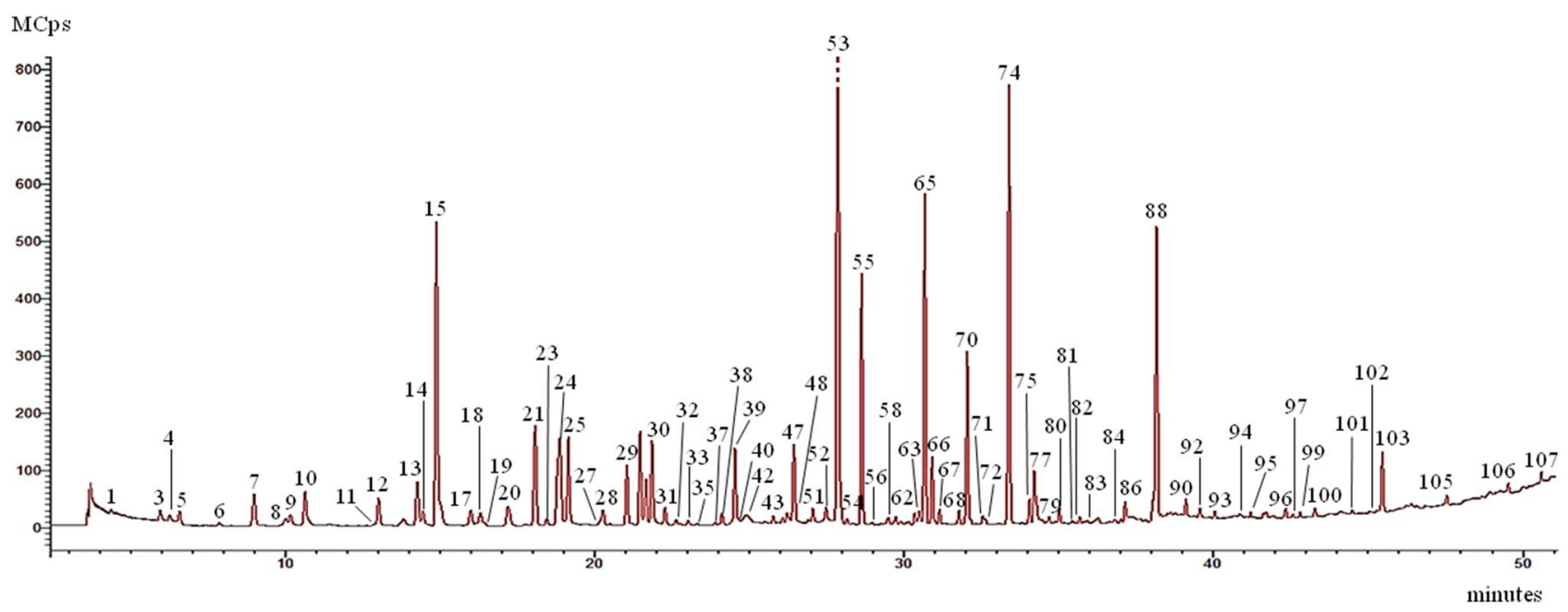

Fig. 1. HS-SPME-GC/MS chromatogram for a Galega Vulgar olive oil sample. For experimental conditions see the text in section 2.2 and 2.3. Peaks are reported in order of elution. The peaks were assigned as in Table 2. 
Table 2

Volatile compounds identified in at least one of the EVOO samples.

\begin{tabular}{|c|c|c|c|c|c|c|c|}
\hline Peak no. ${ }^{a}$ & $\mathrm{RT}^{\mathrm{b}}(\min )$ & Name & Family $^{\mathrm{c}}$ & Ions $m / z$ & Chemical formula & $\mathrm{O} / \mathrm{C}^{\mathrm{d}}$ & $\mathrm{H} / \mathrm{C}^{\mathrm{e}}$ \\
\hline 1 & 4.38 & 1-pentene & Alk & $42 / 55 / 41$ & $\mathrm{C}_{5} \mathrm{H}_{10}$ & 0.00 & 2.00 \\
\hline 2 & 4.79 & Hexane & Alk & $57 / 41 / 56$ & $\mathrm{C}_{6} \mathrm{H}_{14}$ & 0.00 & 2.33 \\
\hline 3 & 6.00 & 1,3-pentadiene* & Alk & $67 / 68 / 53$ & $\mathrm{C}_{5} \mathrm{H}_{8}$ & 0.00 & 1.60 \\
\hline 4 & 6.30 & Cyclopropaneethanol & Alc & $67 / 41 / 40$ & $\mathrm{C}_{5} \mathrm{H}_{10} \mathrm{O}$ & 0.20 & 2.00 \\
\hline 5 & 6.64 & Acetaldehyde & Ald & $44 / 43 / 42$ & $\mathrm{C}_{2} \mathrm{H}_{4} \mathrm{O}$ & 0.50 & 2.00 \\
\hline 6 & 7.94 & Dimethyl sulfide & Sul & $62 / 47 / 45$ & $\mathrm{C}_{2} \mathrm{H}_{6} \mathrm{~S}$ & 0.00 & 3.00 \\
\hline 7 & 9.06 & Octane & Alk & $43 / 41 / 85 / 57$ & $\mathrm{C}_{8} \mathrm{H}_{18}$ & 0.00 & 2.25 \\
\hline 8 & 10.23 & 2-propanone & Ket & $43 / 58$ & $\mathrm{C}_{3} \mathrm{H}_{6} \mathrm{O}$ & 0.33 & 2.00 \\
\hline 9 & 10.72 & Acetic acid, methyl ester & Str & $43 / 74 / 42 / 59$ & $\mathrm{C}_{3} \mathrm{H}_{6} \mathrm{O}_{2}$ & 0.67 & 2.00 \\
\hline 10 & 10.85 & 1-octene & Alk & $41 / 43 / 55 / 70$ & $\mathrm{C}_{8} \mathrm{H}_{16}$ & 0.00 & 2.00 \\
\hline 11 & 13.00 & 1,3-propanediol, 2,2-bis(hydroxymethyl)- & Alc & $43 / 41 / 57 / 70$ & $\mathrm{C}_{5} \mathrm{H}_{12} \mathrm{O}_{4}$ & 0.80 & 2.40 \\
\hline 12 & 13.04 & Ethyl acetate & Alc & $43 / 61 / 70$ & $\mathrm{C}_{4} \mathrm{H}_{8} \mathrm{O}_{2}$ & 0.50 & 2.00 \\
\hline 13 & 14.39 & Butanal, 2-methyl- & Ald & $41 / 57 / 58$ & $\mathrm{C}_{5} \mathrm{H}_{10} \mathrm{O}$ & 0.20 & 2.00 \\
\hline 14 & 14.56 & Butanal, 3-methyl- & Ald & $41 / 44 / 43 / 58$ & $\mathrm{C}_{5} \mathrm{H}_{10} \mathrm{O}$ & 0.20 & 2.00 \\
\hline 15 & 14.99 & Propanoic acid, 2-hydroxy- & Acid & $45 / 43$ & $\mathrm{C}_{3} \mathrm{H}_{6} \mathrm{O}_{3}$ & 1.00 & 2.00 \\
\hline 16 & 15.70 & Hexane, 1-methoxy- & Other & $45 / 56 / 41 / 69$ & $\mathrm{C}_{7} \mathrm{H}_{16} \mathrm{O}$ & 0.14 & 2.29 \\
\hline 17 & 16.11 & 3-ethyl-1,5-octadiene* & Alk & $41 / 69 / 68$ & $\mathrm{C}_{10} \mathrm{H}_{18}$ & 0.00 & 1.80 \\
\hline 18 & 16.43 & 3-ethyl-1,5-octadiene* & Alk & $41 / 69 / 68$ & $\mathrm{C}_{10} \mathrm{H}_{18}$ & 0.00 & 1.80 \\
\hline 19 & 16.56 & Propanoic acid, 2-methyl-, ethyl ester & Str & $43 / 71 / 41$ & $\mathrm{C}_{6} \mathrm{H}_{12} \mathrm{O}_{2}$ & 0.33 & 2.00 \\
\hline 20 & 17.39 & Pentanal & Ald & $44 / 41 / 57$ & $\mathrm{C}_{5} \mathrm{H}_{10} \mathrm{O}$ & 0.20 & 2.00 \\
\hline 21 & 18.21 & 3-ethyl-1,5-octadiene* & Alk & $41 / 69 / 67$ & $\mathrm{C}_{10} \mathrm{H}_{18}$ & 0.00 & 1.80 \\
\hline 22 & 18.44 & 3-hexene, 1-methoxy-* & Other & $45 / 67 / 82$ & $\mathrm{C}_{7} \mathrm{H}_{14} \mathrm{O}$ & 0.14 & 2.00 \\
\hline 23 & 18.58 & Butanoic acid, 2-methyl-, methyl ester & Str & $88 / 57 / 41 / 101$ & $\mathrm{C}_{6} \mathrm{H}_{12} \mathrm{O}_{2}$ & 0.33 & 2.00 \\
\hline 24 & 19.13 & 3-ethyl-1,5-octadiene* & Alk & $41 / 69 / 67$ & $\mathrm{C}_{10} \mathrm{H}_{18}$ & 0.00 & 1.80 \\
\hline 25 & 19.28 & 1-penten-3-one & Ket & $55 / 84 / 56$ & $\mathrm{C}_{5} \mathrm{H}_{8} \mathrm{O}$ & 0.20 & 1.60 \\
\hline 26 & 20.32 & 2-butenal* & Ald & $70 / 41 / 69$ & $\mathrm{C}_{4} \mathrm{H}_{6} \mathrm{O}$ & 0.25 & 1.50 \\
\hline 27 & 20.32 & Toluene & Alk & $91 / 41 / 92$ & $\mathrm{C}_{7} \mathrm{H}_{8}$ & 0.00 & 1.14 \\
\hline 28 & 20.38 & Butanoic acid, 2-methyl-, ethyl ester & Str & $57 / 102 / 41 / 85$ & $\mathrm{C}_{7} \mathrm{H}_{14} \mathrm{O}_{2}$ & 0.29 & 2.00 \\
\hline 29 & 21.16 & 2-octene, 2-methyl-6-methylene- & Alk & $69 / 41 / 67$ & $\mathrm{C}_{10} \mathrm{H}_{18}$ & 0.00 & 1.80 \\
\hline 30 & 21.99 & 2,4-pentadienal & Ald & $44 / 41 / 56$ & $\mathrm{C}_{5} \mathrm{H}_{6} \mathrm{O}$ & 0.20 & 1.20 \\
\hline 31 & 22.40 & 3-pentanol & Alc & $59 / 41 / 55$ & $\mathrm{C}_{5} \mathrm{H}_{12} \mathrm{O}$ & 0.20 & 2.40 \\
\hline 32 & 22.66 & 2-butenal, 2-methyl- & Ald & $55 / 84 / 41$ & $\mathrm{C}_{5} \mathrm{H}_{8} \mathrm{O}$ & 0.20 & 1.60 \\
\hline 33 & 23.04 & 1,4-pentadien-3-ol & Alc & $55 / 83 / 41$ & $\mathrm{C}_{5} \mathrm{H}_{8} \mathrm{O}$ & 0.20 & 1.60 \\
\hline 34 & 23.15 & 4-pentenal & Ald & $55 / 41 / 83$ & $\mathrm{C}_{5} \mathrm{H}_{8} \mathrm{O}$ & 0.20 & 1.60 \\
\hline 35 & 23.45 & 1-butanol, 3-methyl-, acetate & Alc & $43 / 55 / 70$ & $\mathrm{C}_{7} \mathrm{H}_{14} \mathrm{O}_{2}$ & 0.29 & 2.00 \\
\hline 36 & 23.66 & 2-propanol, 1-methoxy- & Alc & $45 / 47$ & $\mathrm{C}_{4} \mathrm{H}_{10} \mathrm{O}_{2}$ & 0.50 & 2.50 \\
\hline 37 & 24.01 & Hydroperoxide, hexyl & Other & $43 / 69 / 41 / 56$ & $\mathrm{C}_{6} \mathrm{H}_{14} \mathrm{O}_{2}$ & 0.33 & 2.33 \\
\hline 38 & 24.26 & 2-pentanal* & Ald & $55 / 83 / 84 / 41$ & $\mathrm{C}_{5} \mathrm{H}_{8} \mathrm{O}$ & 0.20 & 1.60 \\
\hline 39 & 24.66 & 1-penten-3-ol & Alc & $57 / 41 / 55$ & $\mathrm{C}_{5} \mathrm{H}_{10} \mathrm{O}$ & 0.20 & 2.00 \\
\hline 40 & 24.79 & 3-hexenal & Ald & $41 / 69 / 55$ & $\mathrm{C}_{6} \mathrm{H}_{10} \mathrm{O}$ & 0.17 & 1.67 \\
\hline 41 & 24.81 & $p$-xylene & Alk & $91 / 106 / 77$ & $\mathrm{C}_{8} \mathrm{H}_{10}$ & 0.00 & 1.25 \\
\hline 42 & 25.02 & 3-hexenal* & Ald & $41 / 69 / 55$ & $\mathrm{C}_{6} \mathrm{H}_{10} \mathrm{O}$ & 0.17 & 1.67 \\
\hline 43 & 25.63 & Butyl 2-methylbutanoate & Acid & $57 / 85 / 56$ & $\mathrm{C}_{9} \mathrm{H}_{18}$ & 0.00 & 2.00 \\
\hline 44 & 25.74 & 2-butenoic acid, 3-methyl-, methyl ester & Str & $83 / 114 / 55$ & $\mathrm{C}_{6} \mathrm{H}_{10} \mathrm{O}_{2}$ & 0.33 & 1.67 \\
\hline 45 & 25.88 & Dodecane & Alk & $57 / 41 / 43 / 71$ & $\mathrm{C}_{11} \mathrm{H}_{24}$ & 0.00 & 2.18 \\
\hline 46 & 26.23 & Octanoic acid, 7-oxo- & Acid & $43 / 55 / 58$ & $\mathrm{C}_{8} \mathrm{H}_{14} \mathrm{O}_{3}$ & 0.38 & 1.75 \\
\hline 47 & 26.37 & Heptanal & Ald & $41 / 55 / 70$ & $\mathrm{C}_{7} \mathrm{H}_{14} \mathrm{O}$ & 0.14 & 2.00 \\
\hline 48 & 26.57 & 1-butanol, 2-methyl- & Alc & $55 / 41 / 70 / 57$ & $\mathrm{C}_{5} \mathrm{H}_{12} \mathrm{O}$ & 0.20 & 2.40 \\
\hline 49 & 26.74 & $o$-xylene & Alk & $91 / 106 / 77 / 105$ & $\mathrm{C}_{8} \mathrm{H}_{10}$ & 0.00 & 1.25 \\
\hline 50 & 27.05 & Limonene & Terp & $68 / 93 / 67$ & $\mathrm{C}_{10} \mathrm{H}_{16}$ & 0.00 & 1.60 \\
\hline 51 & 27.19 & 2-hexenal* & Ald & $41 / 55 / 83 / 69$ & $\mathrm{C}_{6} \mathrm{H}_{10} \mathrm{O}$ & 0.17 & 1.67 \\
\hline 52 & 27.61 & 2-dodecane* & Alk & $55 / 43 / 56 / 69$ & $\mathrm{C}_{12} \mathrm{H}_{24}$ & 0.00 & 2.00 \\
\hline 53 & 28.01 & 2-hexenal* & Ald & $41 / 55 / 69 / 83$ & $\mathrm{C}_{6} \mathrm{H}_{10} \mathrm{O}$ & 0.17 & 1.67 \\
\hline 54 & 28.10 & 1-pentanol & Alc & $42 / 55 / 41 / 70$ & $\mathrm{C}_{5} \mathrm{H}_{12} \mathrm{O}$ & 0.20 & 2.40 \\
\hline 55 & 28.78 & 1,3,6-octatriene, 3,7-dimethyl-* & Alk & $93 / 91 / 79$ & $\mathrm{C}_{10} \mathrm{H}_{16}$ & 0.00 & 1.60 \\
\hline 56 & 29.11 & 3-octanone & Ket & $43 / 57 / 72 / 99$ & $\mathrm{C}_{8} \mathrm{H}_{16} \mathrm{O}$ & 0.13 & 2.00 \\
\hline 57 & 29.39 & Acetic acid, hexyl ester & Str & $43 / 56 / 69$ & $\mathrm{C}_{8} \mathrm{H}_{16} \mathrm{O}_{2}$ & 0.25 & 2.00 \\
\hline 58 & 29.63 & Butanedioic acid, phenyl- & Acid & $104 / 78 / 69$ & $\mathrm{C}_{10} \mathrm{H}_{10} \mathrm{O}_{4}$ & 0.40 & 1.00 \\
\hline 59 & 29.79 & Tridecane & Alk & $57 / 43 / 41 / 71$ & $\mathrm{C}_{13} \mathrm{H}_{28}$ & 0.00 & 2.15 \\
\hline 60 & 30.05 & Benzene, 1-methyl-4-(1-methylethyl)- & Alk & $119 / 44 / 91$ & $\mathrm{C}_{10} \mathrm{H}_{14}$ & 0.00 & 1.40 \\
\hline 61 & 30.30 & 2-octanone & Ket & $43 / 58 / 45$ & $\mathrm{C}_{8} \mathrm{H}_{16} \mathrm{O}$ & 0.13 & 2.00 \\
\hline 62 & 30.49 & Octanal & Ald & $41 / 43 / 57$ & $\mathrm{C}_{8} \mathrm{H}_{16} \mathrm{O}$ & 0.13 & 2.00 \\
\hline 63 & 30.66 & 4-penten-2-ol & Alc & $45 / 43 / 88$ & $\mathrm{C}_{5} \mathrm{H}_{10} \mathrm{O}$ & 0.20 & 2.00 \\
\hline 64 & 30.77 & 2-penten-1-ol, (E)- & Alc & $57 / 41 / 44$ & $\mathrm{C}_{5} \mathrm{H}_{10} \mathrm{O}$ & 0.20 & 2.00 \\
\hline 65 & 30.81 & Cyclohexane, 2-ethenyl-1,1-dimethyl-3-methylene- & Alk & $69 / 41 / 79$ & $\mathrm{C}_{11} \mathrm{H}_{18}$ & 0.00 & 1.64 \\
\hline 66 & 31.06 & 2-penten-1-ol, (Z)- & Alc & $57 / 41 / 68$ & $\mathrm{C}_{5} \mathrm{H}_{10} \mathrm{O}$ & 0.20 & 2.00 \\
\hline 67 & 31.30 & 3-hexen-1-ol, acetate* & Alc & $67 / 43 / 82 / 55$ & $\mathrm{C}_{8} \mathrm{H}_{14} \mathrm{O}_{2}$ & 0.25 & 1.75 \\
\hline 68 & 31.83 & 2-hexenyl, acetate & Other & $43 / 67 / 41 / 82$ & $\mathrm{C}_{8} \mathrm{H}_{14} \mathrm{O}_{2}$ & 0.25 & 1.75 \\
\hline 69 & 32.15 & 2-heptenal* & Ald & $41 / 55 / 83$ & $\mathrm{C}_{7} \mathrm{H}_{12} \mathrm{O}$ & 0.14 & 1.71 \\
\hline 70 & 32.30 & 5-hepten-2-one, 6-methyl- & Ket & $43 / 41 / 55 / 69$ & $\mathrm{C}_{8} \mathrm{H}_{14} \mathrm{O}$ & 0.13 & 1.75 \\
\hline 71 & 32.69 & 3-hexen-1-ol & Alc & $41 / 67 / 69 / 55$ & $\mathrm{C}_{6} \mathrm{H}_{12} \mathrm{O}$ & 0.17 & 2.00 \\
\hline 72 & 32.78 & Dioxa-1,6-spiro[4.5]decane & Other & $87 / 84 / 41 / 55$ & $\mathrm{C}_{8} \mathrm{H}_{14} \mathrm{O}_{2}$ & 0.25 & 1.75 \\
\hline 73 & 32.94 & Benzene, methoxy- & Other & $108 / 78 / 65$ & $\mathrm{C}_{7} \mathrm{H}_{8} \mathrm{O}$ & 0.14 & 1.14 \\
\hline 74 & 33.54 & 3-hexen-1-ol* & Alc & $67 / 41 / 55 / 82$ & $\mathrm{C}_{6} \mathrm{H}_{12} \mathrm{O}$ & 0.17 & 2.00 \\
\hline
\end{tabular}


Table 2 (continued)

\begin{tabular}{|c|c|c|c|c|c|c|c|}
\hline Peak no. ${ }^{a}$ & $\mathrm{RT}^{\mathrm{b}}(\min )$ & Name & Family ${ }^{c}$ & Ions $m / z$ & Chemical formula & $\mathrm{O} / \mathrm{C}^{\mathrm{d}}$ & $\mathrm{H} / \mathrm{C}^{\mathrm{e}}$ \\
\hline 75 & 34.20 & 2-hexen-1-ol* & Alc & $57 / 41 / 67 / 82$ & $\mathrm{C}_{6} \mathrm{H}_{12} \mathrm{O}$ & 0.17 & 2.00 \\
\hline 76 & 34.36 & Nonanal & Ald & $41 / 43 / 55 / 70$ & $\mathrm{C}_{9} \mathrm{H}_{18} \mathrm{O}$ & 0.11 & 2.00 \\
\hline 77 & 34.44 & Ethanol, 2-butoxy- & Alc & $57 / 41 / 45 / 87$ & $\mathrm{C}_{6} \mathrm{H}_{14} \mathrm{O}$ & 0.17 & 2.33 \\
\hline 78 & 34.84 & 2,4-eexadienal* & Ald & $81 / 41 / 53 / 67$ & $\mathrm{C}_{6} \mathrm{H}_{8} \mathrm{O}$ & 0.17 & 1.33 \\
\hline 79 & 35.01 & 2,5-dimethyl-2,5-dihydroxyhexane & Other & $43 / 113 / 70 / 55$ & $\mathrm{C}_{8} \mathrm{H}_{18} \mathrm{O}_{2}$ & 0.25 & 2.25 \\
\hline 80 & 35.15 & 2,4-hexadienal* & Ald & $81 / 53 / 67$ & $\mathrm{C}_{6} \mathrm{H}_{8} \mathrm{O}$ & 0.17 & 1.33 \\
\hline 81 & 35.58 & 7-hepten-3-ol & Alc & $57 / 72 / 41 / 43$ & $\mathrm{C}_{7} \mathrm{H}_{16} \mathrm{O}$ & 0.14 & 2.29 \\
\hline 82 & 35.69 & 1-heptanol & Alc & $70 / 41 / 56 / 42$ & $\mathrm{C}_{7} \mathrm{H}_{18} \mathrm{O}$ & 0.14 & 2.57 \\
\hline 83 & 35.82 & 2 -octenal & Ald & $55 / 41 / 70$ & $\mathrm{C}_{8} \mathrm{H}_{14} \mathrm{O}$ & 0.13 & 1.75 \\
\hline 84 & 36.98 & Oxirane, [[(2-ethylhexyl)oxy]methyl]- & Other & $57 / 41 / 70 / 83$ & $\mathrm{C}_{11} \mathrm{H}_{22} \mathrm{O}_{2}$ & 0.18 & 2.00 \\
\hline 85 & 36.99 & 1-hexanol, 2-ethyl- & Alc & $57 / 41 / 43 / 70$ & $\mathrm{C}_{8} \mathrm{H}_{8} \mathrm{O}$ & 0.13 & 2.25 \\
\hline 86 & 37.02 & 2,4-heptadienal* & Ald & $81 / 53 / 79$ & $\mathrm{C}_{7} \mathrm{H}_{10} \mathrm{O}$ & 0.14 & 1.43 \\
\hline 87 & 37.16 & Cyclohexane, 1,1-di-hydroxymethyl- & Other & $95 / 96 / 81 / 67$ & $\mathrm{C}_{8} \mathrm{H}_{16} \mathrm{O}_{2}$ & 0.25 & 2.00 \\
\hline 88 & 38.31 & Copaene & Terp & $119 / 105 / 161$ & $\mathrm{C}_{15} \mathrm{H}_{24}$ & 0.00 & 1.60 \\
\hline 89 & 39.18 & Trans- $\alpha$-bergamotene & Terp & $93 / 119 / 43 / 69$ & $\mathrm{C}_{15} \mathrm{H}_{24}$ & 0.00 & 1.60 \\
\hline 90 & 39.25 & 1-octanol & Alc & $56 / 55 / 41 / 70$ & $\mathrm{C}_{8} \mathrm{H}_{20} \mathrm{O}$ & 0.13 & 2.50 \\
\hline 91 & 39.54 & 2-nonenal* & Ald & $43 / 70 / 60$ & $\mathrm{C}_{9} \mathrm{H}_{16} \mathrm{O}$ & 0.11 & 1.78 \\
\hline 92 & 39.72 & Methylcinnamic aldehyde & Ald & $45 / 43 / 77 / 105$ & $\mathrm{C}_{10} \mathrm{H}_{10} \mathrm{O}$ & 0.10 & 1.00 \\
\hline 93 & 40.00 & $n$-valeric anhydride & Acid & $57 / 85 / 86$ & $\mathrm{C}_{10} \mathrm{H}_{8} \mathrm{O}_{3}$ & 0.30 & 0.80 \\
\hline 94 & 40.99 & Dimethyl sulfoxide & Sul & $63 / 78 / 45$ & $\mathrm{C}_{2} \mathrm{H}_{6} \mathrm{OS}$ & 0.50 & 3.00 \\
\hline 95 & 41.24 & 2-octen-1-ol* & Alc & $57 / 41 / 55$ & $\mathrm{C}_{8} \mathrm{H}_{16} \mathrm{O}$ & 0.13 & 2.00 \\
\hline 96 & 42.48 & 1-nonanol & Alc & $55 / 56 / 69$ & $\mathrm{C}_{9} \mathrm{H}_{20} \mathrm{O}$ & 0.11 & 2.22 \\
\hline 97 & 42.74 & Benzoic acid, methyl ester & Str & $105 / 77 / 136$ & $\mathrm{C}_{8} \mathrm{H}_{8} \mathrm{O}_{2}$ & 0.25 & 1.00 \\
\hline 98 & 42.83 & trans- $\beta$-farnesene & Terp & $69 / 41 / 91$ & $\mathrm{C}_{15} \mathrm{H}_{24}$ & 0.00 & 1.60 \\
\hline 99 & 42.96 & 2 -decenal* & Ald & $41 / 55 / 70$ & $\mathrm{C}_{10} \mathrm{H}_{18} \mathrm{O}$ & 0.10 & 1.80 \\
\hline 100 & 43.45 & Benzene, [(methoxymethoxy)methyl]- & Other & $91 / 45 / 92 / 120$ & $\mathrm{C}_{9} \mathrm{H}_{12} \mathrm{O}_{2}$ & 0.22 & 1.33 \\
\hline 101 & 44.65 & $\beta$-curcumene & Terp & $45 / 93 / 119$ & $\mathrm{C}_{15} \mathrm{H}_{24}$ & 0.00 & 1.60 \\
\hline 102 & 45.44 & $\alpha$-farnesene & Terp & $45 / 93 / 69 / 91$ & $\mathrm{C}_{15} \mathrm{H}_{24}$ & 0.00 & 1.60 \\
\hline 103 & 45.62 & $\alpha$-muurolene & Terp & $105 / 161 / 91$ & $\mathrm{C}_{15} \mathrm{H}_{24}$ & 0.00 & 1.60 \\
\hline 104 & 46.50 & 7-formylbicyclo[4.1.0]heptane & Other & $81 / 80 / 79 / 124$ & $\mathrm{C}_{8} \mathrm{H}_{12} \mathrm{O}$ & 0.13 & 1.50 \\
\hline 105 & 47.73 & Benzoic acid, 2-hydroxy-, methyl ester & Str & $120 / 92 / 152$ & $\mathrm{C}_{8} \mathrm{H}_{8} \mathrm{O}_{3}$ & 0.38 & 1.00 \\
\hline 106 & 49.73 & 1-phenylpropane-1,2-diol & Alc & 79/108/77/107 & $\mathrm{C}_{9} \mathrm{H}_{12} \mathrm{O}_{2}$ & 0.22 & 1.33 \\
\hline 107 & 50.80 & Benzenemethanol, $\alpha$-methyl- & Alc & $91 / 92 / 65 / 122$ & $\mathrm{C}_{8} \mathrm{H}_{10} \mathrm{O}$ & 0.13 & 1.25 \\
\hline
\end{tabular}

a Numbers correspond to chromatogram present in Fig. 1.

b Retention time (minutes); * Isomer compounds.

c Alk-linear and branched alkyl, Alc-alcohol, Sul-sulphur compounds, Ket-ketones, Str-sterol, Ald-aldehyde, and Terp-terpenoids.

d Oxygen/carbon ratio.

e Hydrogen/carbon ratio.

compounds and terpenoids, as reported by other authors [4,5,25]. A typical chromatogram of a monovarietal EVOO is shown in Fig. 1. Additionally, the results showed that the differences between calculated and reported LRI has an average variation of approximately 50 units, which agrees with literature [26]. Table 2 lists all compounds detected in at least one of the studied olive oils, showing also other relevant parameters for their characterization, such as retention time, chemical family and chemical formula, characteristic fragmentation pattern (ions $m / z$ ), oxygen/carbon ratio and hydrogen/carbon ratio.

The semi-quantitative data provided by the chromatograms (Table 2) was used to build up the surface density plots (Fig. 2A-I), which display a series of clusters of peaks corresponding to the major structural domains of the olive oil volatiles. Nevertheless, the amount of volatile compounds or specific chemical families are linked not only to the type of olive cultivar, but also the agronomic technique, pedoclimatic characteristics and extraction conditions [27,28]. The volatile compounds of all olive oil varieties studied in this work belong mainly to the family of aldehydes, terpenes and ketones. Ketone derivatives are the most abundant compounds in all olive oil varieties, which is in agreement with other previous works $[10,29,30]$. Nevertheless, there were conspicuous compositional differences among varieties, which are reflected in the proportions of the other chemical families. A remarkable proportion of branched alkyl compounds and a low proportion of acidic compounds and sterols characterized the Galega Vulgar variety (Fig. 2A). The Madural variety (Fig. 2B) is composed of a high proportion of alcohols but a low proportion of alkyl compounds (linear and branched). Furthermore, it showed an absence of acidic compounds. The composition of volatiles of Cobrançosa (Fig. 2C) displayed remarkable proportion of branched alkyl compounds, but not of the linear ones. The Carrasquenha variety (Fig. 2D) is made up of a medium contribution of alkyl compounds, mostly the branched type, while Blanqueta (Fig. 2E) showed a high proportion of linear alkyl compounds, as well as acid compounds. The Picual variety (Fig. 2F) had a high proportion of alcohols and linear alkyl compounds, with little contribution from branched alkyl compounds. The volatile compounds of the Arbequina variety (Fig. $2 \mathrm{G}$ ) are concentrated mainly in the ATK (aldehydes, terpenes and ketones) region, not showing a noteworthy contribution from the rest of the families. Cordovil de Serpa (Fig. $2 \mathrm{H}$ ) is highly influenced by linear alkyl compounds, showing also a remarkable contribution of alcohol and acid compounds. Finally, the Verdeal Alentejana variety (Fig. 2I) is composed mainly of linear alkyl and acid compounds.

\subsection{Chemometric analysis}

\subsubsection{Discriminant analysis}

The results of linear discriminant analysis is represented as a scatter diagram in Fig. 3. This analysis was performed to make a classifier model from information regarding the known class, in this case olive oil varieties, and a data matrix (chromatographic data). This statistic tool is applied for pattern recognition by the obtaining of a classifier among classes, which is used to define the best separate class direction. The predictive ability of LDA was $100 \%$, which indicates that the model generated by volatile compounds (chromatographic data) is correct to separate different olive varieties [18]. In fact, amongst the 82 observations used to fit the model, 82 or $100 \%$ were correctly classified (Table S3). Once the existence of a significant $(P<0.05)$ relationship among different olive oil varieties and the chemical composition of 
A
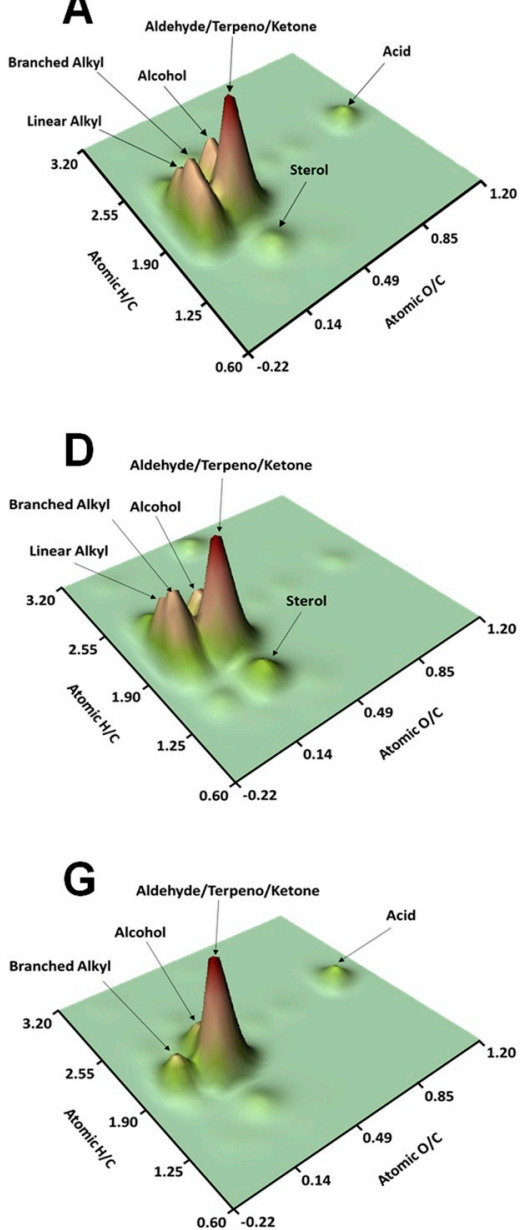

B
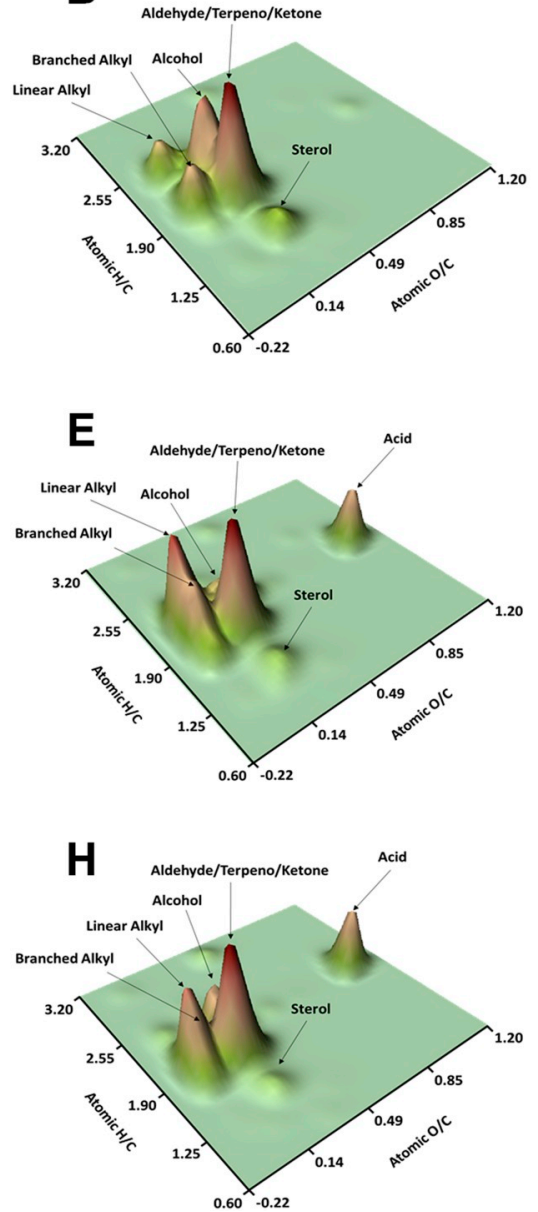
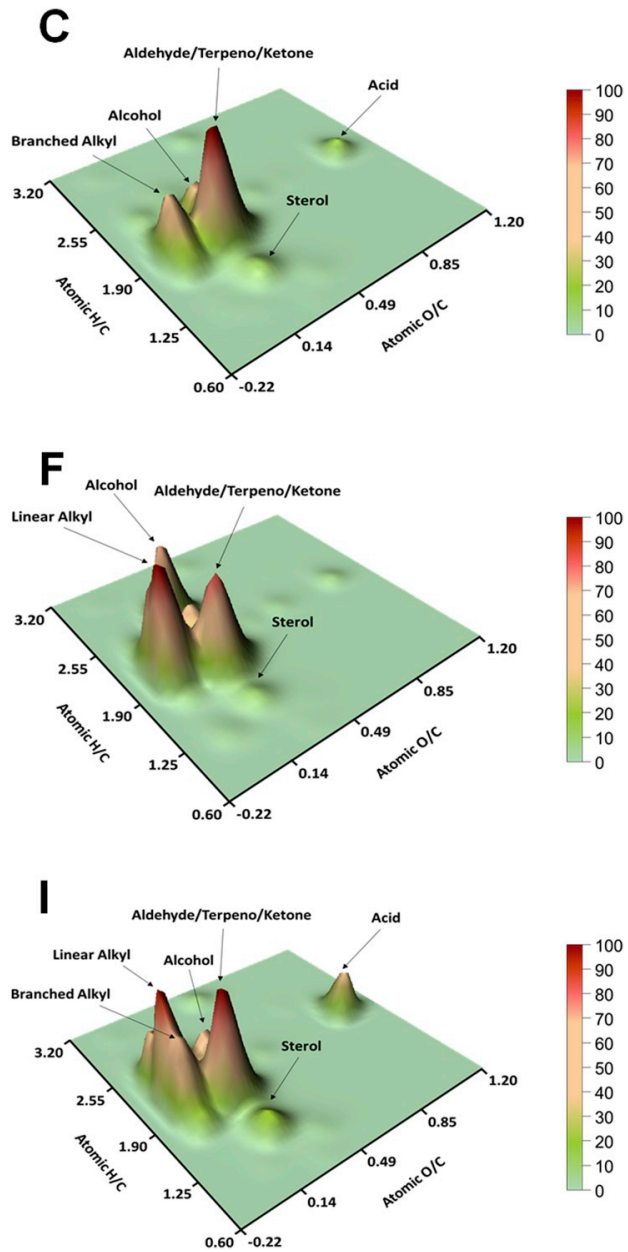

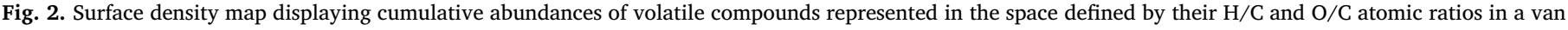

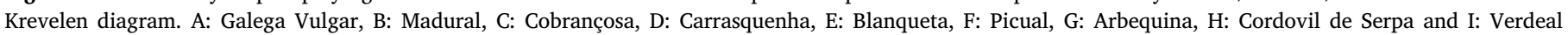
Alentejana.

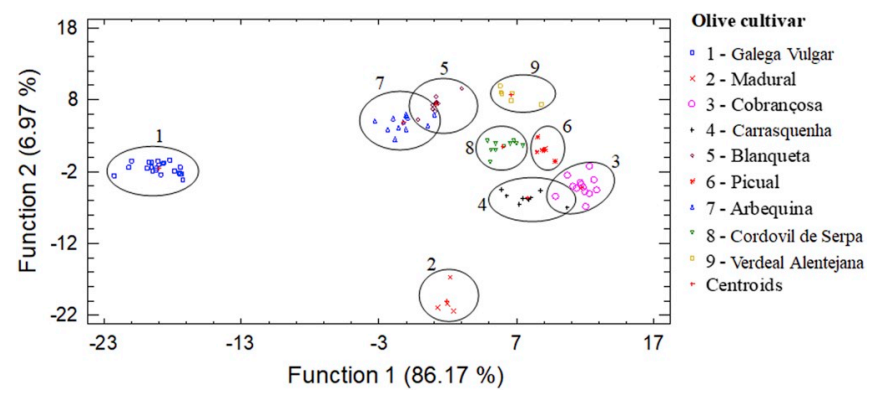

Fig. 3. Discriminant analysis plot of 82 olive oil samples of 9 varieties. All 107. Chromatographic peak areas were employed as independent variables.

volatile compounds is established, the next step is to describe the molecular constituents that are characteristics of each olive oil variety.

\subsubsection{Selection of discriminant markers for each olive oil variety}

For the determination of the molecular markers of each olive oil variety, the magnitude of the main families of compounds was compared using van Krevelen diagrams. For this, a subtraction was done between the map of densities corresponding to the average molecular composition of all the varieties (model sample) and that corresponding to each average of the individual varieties. This subtraction procedure reveals areas where some families of compounds are predominant (blue) or not (red). (Fig. 4A-I). Several researchers [21,23] have used this type of graphical-statistical analysis (subtraction) to determine molecular biomarkers, although in different matrices. A study was carried out using the Student's $t$ test to determine the regions significantly different to $95 \%(P<0.05)$. The significantly different compounds of each variety (Table S2) were plotted on the van Krevelen diagram marked with contours (Fig. 4A-I) superimposed by the subtraction diagrams. The coincidence of areas of the contour plot (significantly different compounds $P<0.05$ ) with subtraction map areas indicates the compounds that are significantly characteristic of each variety of olive oil.

The Galega Vulgar variety (Fig. 4A) is characterized by having a very low concentration of acid type compounds, as well as compounds belonging to the group of ATK and sterols. The compounds belonging to the family of alcohols had a great importance within the Madural variety (Fig. 4B). Further, in this variety there was a decrease of branched alkyl compounds with respect to the sample model (average of the samples). The Cobrançosa variety (Fig. 4C) showed a concentration of ATK compounds higher than the model, but lower than linear chain alkyl compounds. The Carrasquenha variety (Fig. 4D) is characterized by the enormous presence of sulphur compounds, sterols and linear alkyls. In contrast, a concentration of ATK compounds and alcohols lower than the model was observed for the Blanqueta variety (Fig. 4E). The Picual variety (Fig. 4F) presented a high concentration of volatile alcohol, but low ATK compounds and sterols with respect to the model sample. The absence or low proportion of alkyl compounds (linear and branched) was characteristic of the Arbequina variety 
A

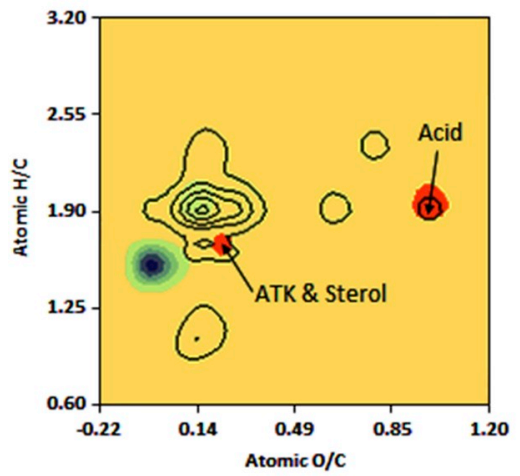

D

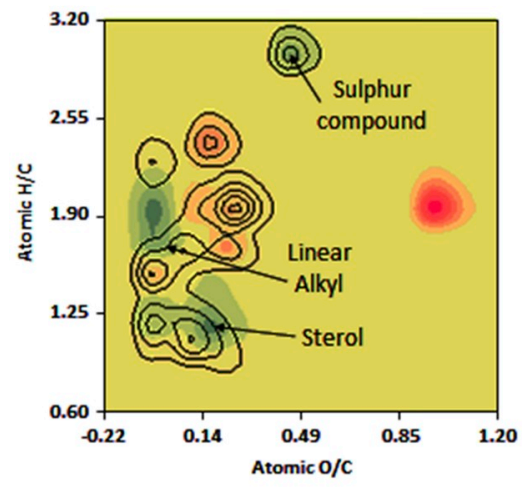

G

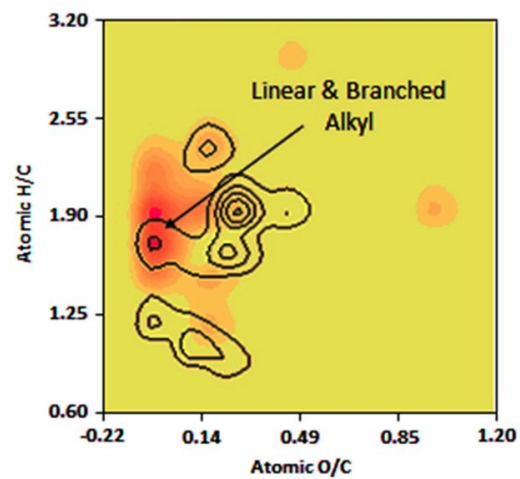

B

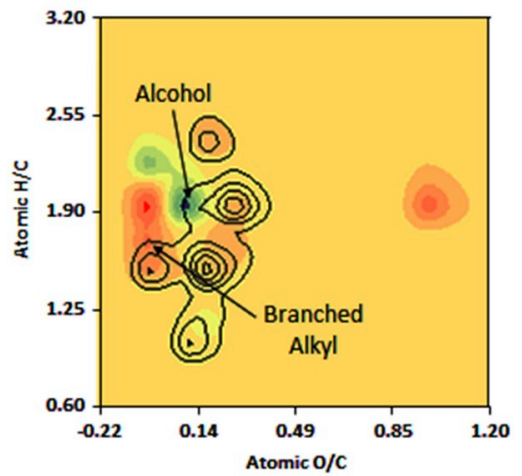

$\mathbf{E}$

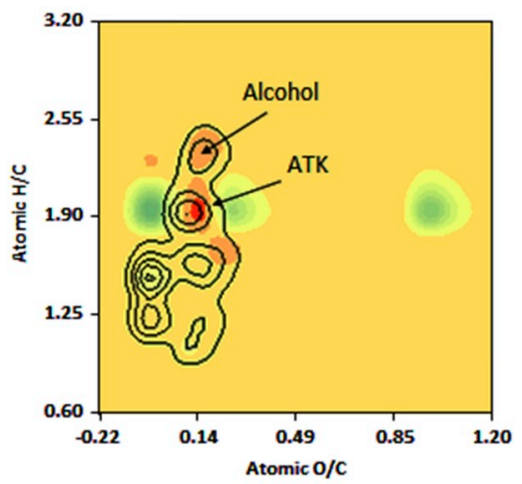

H

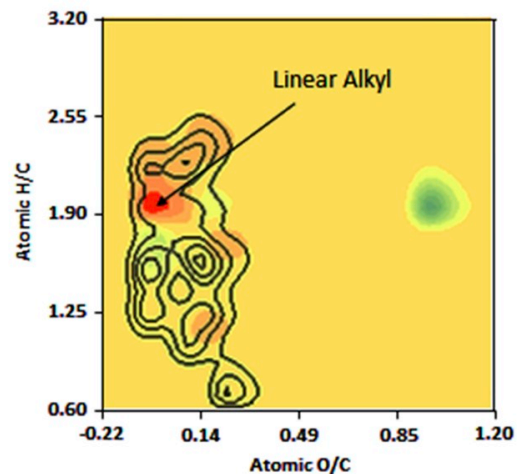

C

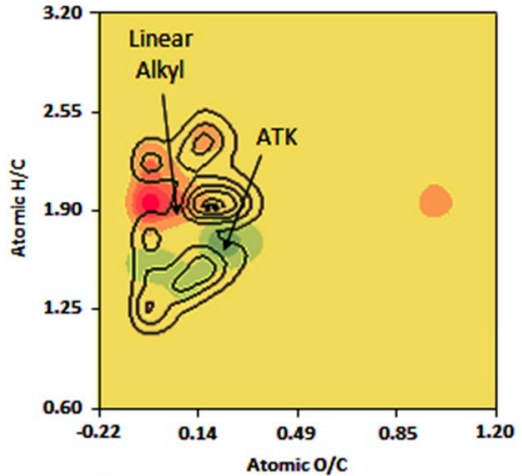

$\mathbf{F}$

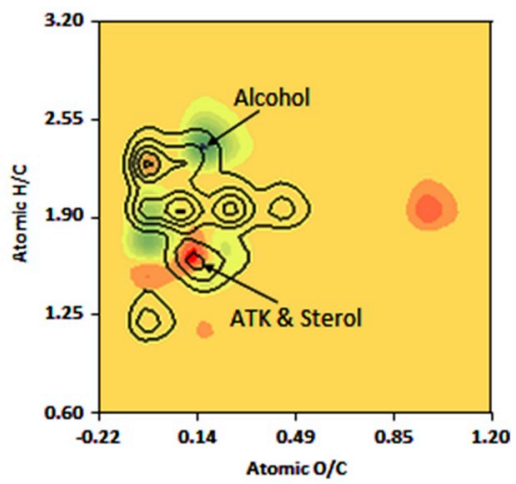

I

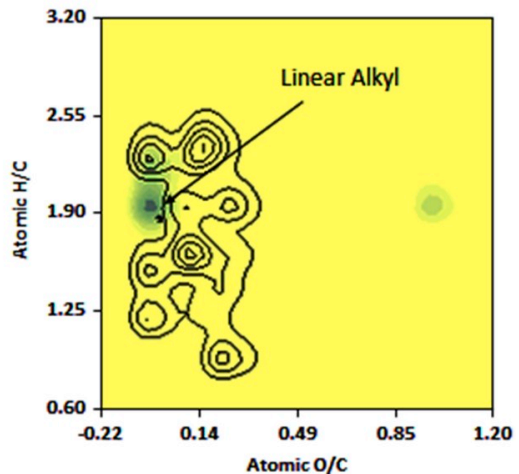

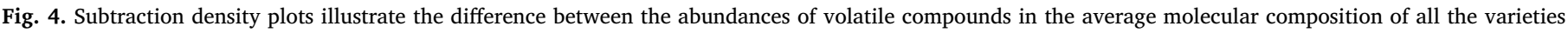

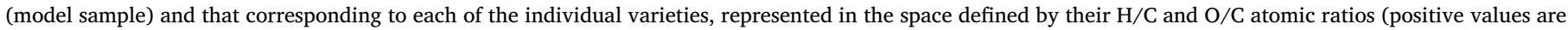

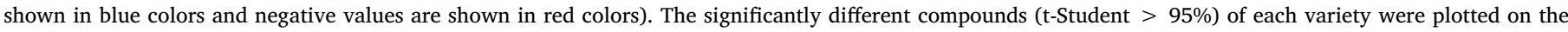

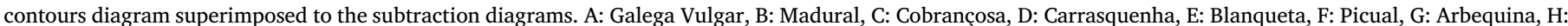

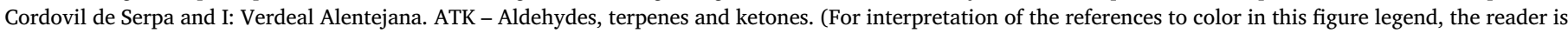
referred to the Web version of this article.)

(Fig. 4G). Finally, linear alkyl compounds characterized the Cordovil de Serpa and Verdeal Alentejana varieties. However, their concentrations were different according to the olive oil variety. Cordovil de Serpa (Fig. $4 \mathrm{H}$ ) variety had a lower concentration than the model sample, while Verdeal Alentejana one (Fig. 4I) displayed an opposite behavior.

\section{Conclusions}

In this study HS-SPME-GC/MS was used to assess the volatile profile of monovarietal EVOO produced with different varieties of olives, allowing the identification and semi-quantification of a total of 107 compounds belonging essentially to the chemical classes of esters, alcohols, aldehydes, acids, ketones, linear and branched alkyl sulphur compounds and terpenoids.
In addition, the combination of statistical analysis (Student's $t$ test) and graphical-tools (van Krevelen diagram) represent a fast and functional method to classify and describe EVOO from different varieties. Furthermore, 3D van Krevelen diagrams proved to be a good tool for interpretation and visualization of complex data, such as the ones related to the volatile compounds of varietal olive oils and represents a step forward in the analysis of complex matrices using mass spectrometry data. This tool shows a huge potential to be further used to identify olive oils varietal origin.

In overall, this study contributes to increase the knowledge of volatile profile of EVOO produced with different varieties from the Alentejo region, enabling to ascribe some molecular markers related to the volatile profile (in terms of chemical families) of each olive oil variety. 


\section{Acknowledgments}

This work was funded by European Regional Development Fund (FEDER) and National Funds through Foundation for Science and Technology (FCT) under Project "Por3O - Portuguese Olive Oil Omics for traceability and authenticity - PTDC/AGRPRO/2003/2014, by National Funds through FCT - Foundation for Science and Technology under the Projects UID/AGR/00115/2019 and FCT/MCTES (UID/QUI/ 50006/2019). Raquel Garcia thanks the Fundação para a Ciência e Tecnologia (FCT) for a post-doctoral research fellowship (SFRH/BPD/ 109912/2015).

Nuno Martins acknowledges French National Agency for Research (ANR-15-ARM2-0002-04) supported by the European Union's Seventh Framework Programme for research, technological development and demonstration under grant agreement number 618127 (ARIMNet2).

\section{References}

[1] N.B. Ray, K.D. Hilsabeck, T.C. Karagiannis, D.E. McCord, Chapter 36 - Bioactive Olive Oil Polyphenols in the Promotion of Health in the Role of Functional Food Security in Global Health, (2019), pp. 623-663.

[2] D. Boskou, Olive Oil: Chemistry and Technology, second ed., Academic Press and AOCS Press, Thessaloniki, Hellas, 2006.

[3] M.D.R. Gomes da Silva, A.M. Freitas, M.J. Cabrita, R. Garcia, Olive oil composition: volatile compounds, in: D. Boskou (Ed.), Olive Oil - Constituents, Quality, Health Properties and Bioconversions, InTech, Croatia, 2012.

[4] C.M. Kalua, M.S. Allen, D.R. Bedgood Jr., A.G. Bishop, P.D. Prenzler, K. Robards, Olive oil volatile compounds, flavour development and quality: a critical review, Food Chem. 100 (2007) 273-286 https://doi.org/10.1016/j.foodchem.2005.09. 059.

[5] T. Cecchi, B. Alfei, Volatile profiles of Italian monovarietal extra virgin olive oils via HS-SPME-GC-MS: newly identified compounds, flavors molecular markers, and terpenic profile, Food Chem. 141 (2013) 2025-2035 https://doi.org/10.1016/j. foodchem.2013.05.090.

[6] M. Cherfaoui, T. Cecchi, S. Keciri, L. Boudriche, Volatile compounds of Algerian extra-virgin olive oils: effects of cultivar and ripening stage, Int. J. Food Prop. 21 (2018) 51-64 https://doi.org/10.1080/10942912.2018.1437627.

[7] S. Vichi, A.I. Castellote, L. Pizzale, L.S. Conte, S. Buxaderas, E. López-Tamames, Analysis of virgin olive oil volatile compounds by headspace solid-phase microextraction coupled to gas chromatography with mass spectrometric and flame ionization detection, J. Chromatogr. A 983 (2003) 19-33 https://doi.org/10.1016/ S0021-9673(02)01691-6.

[8] J.F. Cavalli, X. Fernandez, L. Lizzani-Cuvelier, A.M. Loiseau, Characterization of volatile compounds of French and Spanish virgin olive oils by HS-SPME: identification of quality-freshness markers, Food Chem. 88 (2004) 151-157 https://doi. org/10.1016/j.foodchem.2004.04.003.

[9] A. Bendini, E. Valli, S. Barbieri, T. Gallina, Sensory analysis of virgin olive oil, in: D. Boskou (Ed.), Olive Oil Constituents, Quality, Health Properties and Bioconversions, InTech. Croatia, 2012, pp. 109-130 (pp. 109-130).

[10] F. Peres, L.L. Martins, S. Ferreira-Dias, Influence of enzymes and technology on virgin olive oil composition, Crit. Rev. Food Sci. Nutr. 57 (2017) 3104-3126 https://doi.org/10.1080/10408398.2015.1092107.

[11] M. D'Imperio, M. Gobbino, A. Picanza, S. Costanzo, A. Della Corte, L. Mannina, Influence of harvest method and period on olive oil composition: an NMR and statistical study, J. Agric. Food Chem. 58 (2010) 11043-11051 https://pubs.acs. org/doi/abs/10.1021/jf1026982.

[12] G. Fregapane, M.D. Salvador, Production of superior quality extra virgin olive oil modulating the content and profile of its minor components, Food Res. Int. 54 (2013) 1907-1914 https://doi.org/10.1016/j.foodres.2013.04.022.
[13] A.F. Vinha, F. Ferreres, B.M. Silva, P. Valentão, A. Goncalves, J.A. Pereira, M.B. Oliveira, R.M. Seabra, P.B. Andrade, Phenolic profiles of Portuguese olive fruits (Olea europaea L.): influences of cultivar and geographical origin, Food Chem. 89 (2005) 561-568 https://doi.org/10.1016/j.foodchem.2004.03.012.

[14] L. Cerretani, A. Bendini, A. del Caro, A. Piga, V. Vacca, M.F. Caboni, T.G. Toschi, Preliminary characterisation of virgin olive oils obtained from different cultivars in Sardinia, Eur. Food Res. Technol. 222 (2006) 354-361 https://link.springer.com/ article/10.1007/s00217-005-0088-9.

[15] P. Guedes de Pinho, R.F. Gonc-alves, P. Valentao, D.M. Pereira, R.M. Seabra, P.B. Andrade, M. Sottomayor, Volatile composition of Catharanthus roseus (L.) G. Don using solid-phase microextraction and gas chromatography/mass spectrometry, J. Pharm. Biomed. Anal. 49 (2009) 674-685 https://doi.org/10.1016/j.jpba. 2008.12.032.

[16] E.V. Avramidoul, A.G. Doulis, P.V. Petrakis, Chemometrical and molecular methods in olive oil analysis: a review, J. Food Process. Preserv. 42 (2018) e13770, https:// doi.org/10.1111/jfpp.13770.

[17] A. Cimato, D. Dello Monaco, C. Distante, M. Epifani, P. Siciliano, A.M. Taurino, M. Zuppa, G. Sani, Analysis of single-cultivar extra virgin olive oils by means of an Electronic Nose and HS-SPME/GC/MS methods, Sens. Actuators B Chem. 114 (2006) 674-680 https://doi.org/10.1016/j.snb.2005.06.058.

[18] E. Pouliarekou, A. Badeka, M. Tasioula-Margari, S. Kontakos, F. Longobardi, M.G. Kontominas, Characterization and classification of Western Greek olive oils according to cultivar and geographical origin based on volatile compounds, J. Chromatogr. A 1218 (2011) 7534-7542 https://doi.org/10.1016/j.chroma.2011. 07.081.

[19] D.W. van Krevelen, Graphical-statistical method for the study of structure and reaction processes of coal, Fuel 2 (1950) 269-284.

[20] N.T. Jiménez-Morillo, J.M. de la Rosa, D. Waggoner, G. Almendros, F.J. GonzálezVila, J.A. González-Pérez, Fire effects in the molecular structure of soil organic matter fractions under Quercus suber cover, Catena 145 (2016) 266-273 https:// doi.org/10.1016/j.catena.2016.06.022.

[21] N.T. Jiménez-Morillo, J.A. González-Pérez, G. Almendros, J.M. de la Rosa, D.C. Waggoner, A. Jordán, L.M. Zavala, F.J. González-Vila, G. Patrick, G. Hatcher, Ultra-high resolution mass spectrometry of physical speciation patterns of organic matter in fire-affected soils, J. Environ. Manag. 225 (2018) 139-147 https://doi. org/10.1016/j.jenvman.2018.07.069.

[22] Z. Hernández, G. Almendros, A. Álvarez, T. Figueiredo, P. Carral, Soil carbon stabilization pathways as reflected by the pyrolytic signature of humic acid in agricultural volcanic soils, J. Anal. Appl. Pyrolysis 137 (2019) 14-28 https://doi.org/ 10.1016/j.jaap.2018.10.015

[23] G. Almendros, Z. Hernández, J. Sanz, S. Rodríguez-Sánchez, M.A. JiménezGonzález, J.A. González-Pérez, Graphical statistical approach to soil organic matter resilience using analytical pyrolysis data, J. Chromatogr. A 1533 (2018) 164-173 https://doi.org/10.1016/j.chroma.2017.12.015.

[24] N. Martins, R. Garcia, D. Mendes, A.M. Freitas, M.D.R. Gomes da Silva, M.J. Cabrita, An ancient winemaking technology: exploring the volatile composition of amphora wines, LWT - Food Sci. Technol. (Lebensmittel-Wissenschaft -Technol.) 96 (2018) 288-295 https://doi.org/10.1016/j.lwt.2018.05.048.

[25] R. Aparicio, S.M. Rocha, I. Delgadillo, M.T. Morales, Detection of rancidity defect in virgin olive oil by the Electronic Nose, J. Agric. Food Chem. 48 (2000) 853-860 https://pubs.acs.org/doi/abs/10.1021/jf9814087.

[26] K. Tahri, A.A. Duarte, T. Saidi, M. Bougrini, G. Carvalho, P.A. Ribeiro, M.D.R. Gomes da Silva, D. Mendes, N. El Bari, M. Raposo, B. Bouchikhi, Distinguishment, identification and aroma compounds quantification of Portuguese olive oils based on physicochemical attributes HS-GC/MS analysis and voltammetric electronic tongue, J. Sci. Food Agric. 98 (2017) 681-690 https://doi.org/10 1002/jsfa.8515.

[27] K. Brkić Bubola, O. Koprivnjak, B. Sladonja, D. Škevin, I. Belobrajić, Chemical and sensorial changes of Croatian monovarietal olive oils during ripening, Eur. J. Lipid Sci. Technol. 114 (2012) 1400-1408 https://doi.org/10.1002/ejlt.201200121.

[28] G. Luna, M.T. Morales, R. Aparicio, Characterization of 39 varietal virgin olive oils by their volatile compositions, Food Chem. 98 (2006) 243-252 https://doi.org/10. 1016/j.foodchem.2005.05.069.

[29] F. Angerosa, C. Basti, R. Vito, Virgin olive oil volatile compounds from lipoxygenase pathway and characterization of some Italian cultivars, J. Agric. Food Chem. 47 (1999) 836-839 https://pubs.acs.org/doi/full/10.1021/jf980911g.

[30] F. Angerosa, R. Mostallino, C. Basti, R. Vito, Virgin olive oil odour notes: their relationships with volatile compounds from the lipoxygenase pathway and secoiridoid compounds, Food Chem. 68 (2000) 283-287 https://doi.org/10.1016/S03088146(99)00189-2. 